intravenous antibiotics, surgical debridement and drainage, and airway management.

Corsten and associates' ${ }^{4}$ meta-analysis demonstrated a significant survival advantage in those patients who were treated by a combined drainage of the neck and mediastinum versus cervical drainage alone. The authors recommend a formal thoracotomy to drain the chest. Brunelli and colleagues, ${ }^{5}$ however, recommend a cervicomediastinal drainage (cervical incision to enter the thorax) of the mediastinum for cases of superior mediastinitis, with a formal thoracotomy reserved for those cases in which the DNM involvement extends beyond the carina.

Our surgical approach through a median sternotomy associated with a transverse cervical incision was based on the fact that the patient had a compromised cardiac output and CT findings consistent with a predominant anterior and middle mediastinitis. The posterior and infracarinal mediastinum were less affected by the disease. Our purpose was to decompress the heart along with a proper debridement. Inotropic requirements after surgery were significantly lower.

Abscesses tend to reform in the days after surgery, and a daily cervical wash is necessary to release collections and to collapse forming abscesses. Irrigation and drainage of the mediastinum are conducted daily.

Management of DNM should always be surgical, with broad, extensive, open cervicotomy together with ample mediastinal drainage and associated thoracotomy, if necessary, to ensure the adequate drainage of all mediastinal compartments.

\section{References}

1. Lavini C, Natali P, Morandi U, Dallari S, Bergamini G. Descending necrotizing mediastinitis: diagnosis and surgical treatment. $J$ Cardiovasc Surg (Torino). 2003;44:655-660.

2. Estrera AS, Landay MJ, Grisham JM, Sinn DP, Platt MR. Descending necrotizing mediastinitis. Surg Gynecol Obstet. 1983;157:545-52.

3. Kruyt PM, Boonstra A, Fockens P, Reeders JW, van Lanschot JJ. Descending necrotizing mediastinitis causing pleuroesophageal fistula. Chest. 1996;109:1404-7.

4. Cortsen MJ, Shamji FM, Odell PF, Frederico JA, Laframboise GG, Reid KR, et al. Optimal treatment of descending necrotising mediastinitis. Thorax. 1997;52:702-8.

5. Brunelli A, Sabbatini A, Catalini G, Fianchini A. Descending necrotizing mediastinitis. Arch Otolaryngol Head Neck Surg. 1996;122:1326-9.

\title{
Technique of right single-lung transplantation for idiopathic pulmonary fibrosis using cross-field ventilation
}

\author{
Seth D. Force, MD, ${ }^{a}$ Andres Pelaez, MD, ${ }^{\mathrm{b}}$ David C. Neujahr, MD, ${ }^{\mathrm{b}}$ Allan M. Ramirez, MD, ${ }^{\mathrm{b}}$ William Whitley, MD, \\ Daniel L. Miller, MD, ${ }^{a}$ and E. Clinton Lawrence, MD, ${ }^{b}$ Atlanta, Ga
}

I solated lung ventilation for single or bilateral sequential lung transplantation can be achieved with either a double-lumen endotracheal tube or a single-lumen endotracheal tube with a bronchial blocker. However, situations might arise that call for alternative methods of ventilation until the airway can be stabilized. We present a patient in whom we were unable to achieve isolated lung ventilation using standard techniques but were successful with cross-field ventilation of the nontransplanted lung during a right single-lung transplantation.

\section{Clinical Summary}

A 54-year-old man presented for lung transplantation evaluation with end-stage lung disease caused by pulmonary fibrosis. A chest

From the Divisions of Cardiothoracic Surgery ${ }^{\mathrm{a}}$ and Pulmonary Medicine ${ }^{\mathrm{b}}$ and the Department of Anesthesiology, ${ }^{\mathrm{c}}$ Emory University, Atlanta, Ga.

Received for publication July 24, 2006; accepted for publication Sept 5, 2006.

Address for reprints: Seth D. Force, MD, The Emory Clinic, Rm 2213, 1365 Clifton Rd, NE Atlanta, GA 30324 (E-mail: seth_force@emoryhealthcare.org).

J Thorac Cardiovasc Surg 2007;133:272-3

$0022-5223 / \$ 32.00$

Copyright $\odot 2007$ by The American Association for Thoracic Surgery doi:10.1016/j.jtcvs.2006.09.013 computed tomographic scan performed as part of the transplantation evaluation showed fibrotic lung disease bilaterally and a significant rightward angulation of the trachea (Figure 1). Bronchoscopy confirmed the severe deviation of the trachea and revealed the right upper lobe bronchus and bronchus intermedius to be coming directly off of the trachea. A quantitative ventilationperfusion scan showed that the right lung was receiving $34 \%$ of the overall blood and air flow, and therefore the patient was listed for right single-lung transplantation.

At the time of transplantation, because of the severe deviation of the patient's trachea, the double-lumen endotracheal tube was unable to be positioned properly. Single-lung ventilation was next attempted by placing a single-lumen endotracheal tube down the left main bronchus, but this was also unsuccessful. The singlelumen tube was then positioned in the midtrachea, and a bronchial blocker was placed into the right mainstem bronchus.

The patient was positioned in the left lateral decubitus position and underwent a standard right posterolateral thoracotomy incision. The recipient pneumonectomy was performed after an extensive lysis of adhesions, and the endotracheal tube was then directed down the left main bronchus. However, it was not possible to place the endotracheal balloon completely within the bronchus, and a large part of the balloon was therefore obstructing the distal trachea. The endotracheal tube was then withdrawn into the midtrachea, and a number 7.0 armored tube was then placed 


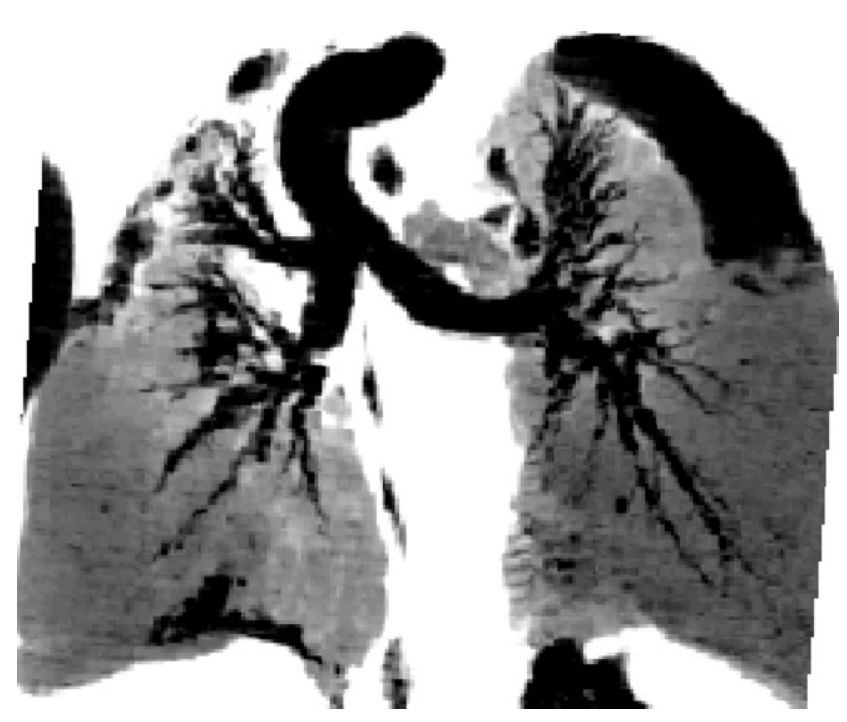

Figure 1. Pretransplantation chest computed tomographic scan.

through the opened right main bronchus and directed down into the left main bronchus. This tube was then connected to a sterile anesthesia circuit and used to ventilate the left lung. The right donor lung was then implanted beginning with the bronchial anastomosis. A 4-0 polydioxanone suture was placed through the donor and recipient bronchi at each membranous-cartilaginous junction and tied. The armored tube was then removed and placed posteriorly through the membranous bronchial anastomosis and directed into the left main bronchus (Figure 2, A). The cartilaginous bronchial anastomosis was then performed, followed by the pulmonary artery and left atrial anastomoses. The lung was then retracted medially, the armored tube was removed, and ventilation was begun through the endotracheal tube positioned in the trachea. The membranous bronchial anastomosis was then performed with a running 4-0 polydioxanone suture. The lung was then reperfused, the chest was closed, and the patient was taken to the intensive care unit in stable condition.

The patient was extubated 3 days later and discharged from the hospital on the 18th postoperative day, saturating $98 \%$ on room air. A postoperative chest computed tomographic scan revealed a healthy lung graft and near-total correction of the tracheal deviation (Figure 2, B).

\section{Discussion}

Knowledge of alternative ventilation techniques is critical for the rare times that more standard techniques fail. Other strategies that could have been used in our case include left lung transplantation, jet ventilation into the left lung, or cardiopulmonary bypass. We chose to transplant the right lung based on the preoperative ventilationperfusion scan and because we believed that we would have

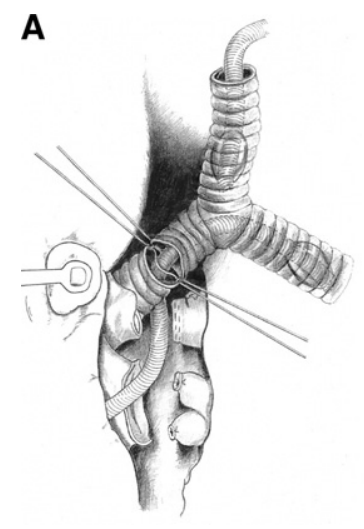

B

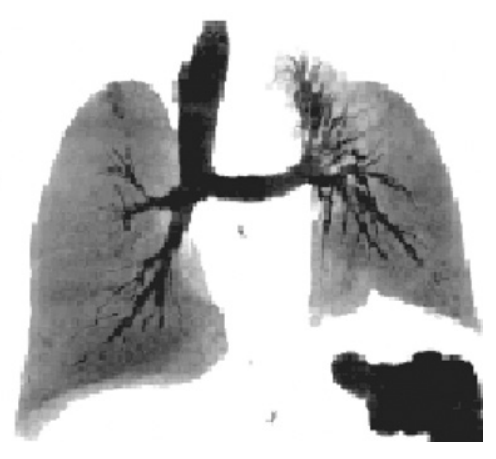

Figure 2. A, Technique of cross-table ventilation during singlelung transplantation. B, Posttransplantation chest computed tomographic scan.

exacerbated the mediastinal shift if we had replaced the left lung with a much more compliant donor lung.

Jet ventilation can be used during pulmonary resections, such as carinal resections, but would not be helpful in the transplant setting because of the inability to maintain isolated lung ventilation. ${ }^{1}$ Cardiopulmonary bypass can also be used electively or urgently for lung transplantation. ${ }^{2}$ However, it has been associated with an increased risk of primary graft dysfunction and would have led to excessive bleeding from the pleural adhesions. ${ }^{3-5}$

In conclusion, we present a patient who underwent a right single-lung transplantation using cross-field left lung ventilation. The operation was performed without difficulty, restored a normal shape to the patient's trachea, and led to the resolution of a left-sided traction pneumothorax. This strategy should be considered among the possible ventilation techniques when lung transplant surgeons encounter difficulties with more standard techniques.

\section{References}

1. Perera ER, Vidic DM, Zivot J. Carinal resection with two highfrequency jet ventilation delivery systems. Can J Anaesth. 1993;40: 59-63.

2. Brusset A, Bennette P, Hatahet Z, Loirat P, Bisson A, Fischler M. Single lung transplantation for pulmonary lymphangiomyomatosis: unexpected need for extracorporeal circulation. Chest. 1995;107:278-82.

3. Sekin Y, Waddell TK, Matte-Martyn A, et al. Risk quantification of early outcome after lung transplantation: donor, recipient, operative and post-transplant parameters. J Heart Lung Transplant. 2004;23:96-104.

4. King RC, Binns AR, Rodriguez F, et al. Reperfusion injury significantly impacts clinical outcome after pulmonary transplantation. Ann Thorac Surg. 2000;69:1681-5.

5. Thabut G, Vinatier I, Stern J, et al. Primary graft failure following lung transplantation. Chest. 2002;121:1876-82. 